\title{
SOME REMARKS ON INDICATRICES OF MEASURABLE FUNCTIONS
}

\author{
MARCIN KYSIAK
}

\begin{abstract}
Summary. We show that for a wide class of $\sigma$-algebras $\mathcal{A}$, indicatrices of $\mathcal{A}$-measurable functions admit the same characterization as indicatrices of Lebesgue-measurable functions. In particular, this applies to functions measurable in the sense of Marczewski.
\end{abstract}

Let $f: X \rightarrow Y$ be a function. The function $s(f): Y \rightarrow$ CARD, defined by the formula $s(f)(y)=\mid f^{-1}[\{y\}]$ is called the (Banach) indicatrix of $f$. For $f, g: X \rightarrow$ $Y$, we say that $f$ is equivalent to $g$, if there exists a bijection $\varphi: X \rightarrow X$ such that $f=g \circ \varphi$. Obviously, this is equivalent to saying that $s(f)=s(g)$.

Morayne and Ryll-Nardzewski show in [5] that a function $f:[0,1] \rightarrow[0,1]$ is equivalent to a Lebesgue-measurable one, if and only if, either $s(f)>0$ on a perfect set $P \subseteq[0,1]$ or there exists $y \in[0,1]$ such that $s(f)(y)=\mathfrak{c}$. In fact, they prove a more general statement. Namely, the same is true for the class of functions which are measurable with respect to the $\sigma$-algebra $\mathcal{A}$ generated by the Borel sets and a $\sigma$-ideal $\mathcal{I}$ with Borel base containing an uncountable set. They also ask about characterization of indicatrices of other important classes of functions.

A characterization of indicatrices of continuous functions was given by Kwiatkowska in [4]. Also, Komisarski, Michalewski and Milewski in [3] characterized (under the

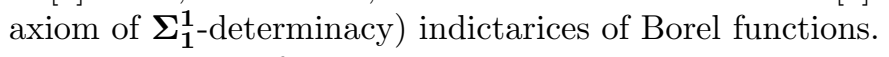

The purpose of this note is to generalize the characterization of Morayne and Ryll-Nardzewski to other classes of measurable functions. We say that a set $X \subseteq$ $[0,1]$ is Marczewski-measurable, if for every perfect set $P \subseteq[0,1]$ there exists a perfect set $Q \subseteq P$ such that $Q \subseteq X$ or $Q \cap X=\emptyset$. Marczewski-measurable sets form a $\sigma$-algebra; a function $f:[0,1] \rightarrow[0,1]$ is Marczewski-measurable, if the preimage of every open set is Marczewski measurable. By Marczewski's theorem (see [7]) this is equivalent to saying that for every perfect set $P$ there exists a perfect set $Q \subseteq P$ such that $f\lceil Q$ is continuous.

We begin with showing that indicatrices of Marczewski-measurable functions admit the same characterization as those of Lebesgue-measurable ones. It is known that the algebra of Marczewski-measurable sets is not of the form considered in [5]. Then we try to isolate the properties of Marczewski-measurable sets and functions used in the proof to obtain a more general result.

For a family of sets $\mathcal{A}$, let $\mathcal{H}(\mathcal{A})=\{A \in \mathcal{A}: \forall B \subseteq A B \in \mathcal{A}\}$. Observe that if $\mathcal{A}$ is a $\sigma$-algebra, then $\mathcal{H}(\mathcal{A})$ is a $\sigma$-ideal.

2000 Mathematics Subject Classification. 28A05, 26A99, 03E15.

Key words and phrases. Indicatrix, Marczewski-measurable function.

The research was done when the author was visiting Institute of Mathematics of the Polish Academy of Sciences. 
The following lemma is a slight modification of an argument from [5]. The main difference is that we do not use the assumption of Borel base of the ideal.

Lemma 1. Let $\mathcal{A}$ be a $\sigma$-algebra containing Bor such that $\mathcal{H}(\mathcal{A})$ contains a set of size c. Let $f:[0,1] \rightarrow[0,1]$ be a function such that $f[[0,1]]$ contains a perfect set. Then $f$ is equivalent to a $\mathcal{A}$-measurable function.

Proof. Let $P$ be a perfect set contained in the image of $f$; we may always assume that $|f[[0,1]] \backslash P|=\mathfrak{c}$. Let $\Psi:[0,1] \rightarrow P$ be a Borel isomorphism and let $M \in \mathcal{H}(\mathcal{A})$ be a set of cardinality $\mathfrak{c}$ such that $|[0,1] \backslash M|=\mathfrak{c}$. Observe that $\Psi$ is $\mathcal{A}$-measurable.

Let $s(f):[0,1] \rightarrow$ CARD be the indicatrix of $f$ and let $\left\{M_{y}: y \in[0,1]\right\}$ be a partition of $M$ such that $\left|M_{y}\right|=s(f)(y)-1$ for $y \in \Psi[[0,1] \backslash M]$ (this is meaningful, because $s(f)(y)>0$ for $y \in P$ and we allow $M_{y}$ to be empty) and $\left|M_{y}\right|=s(f)(y)$ otherwise. Such a partition can be found because for continuum many $y \in[0,1]$ we stipulate that $\left|M_{y}\right|>0$, so $\sum_{y \in[0,1]}\left|M_{y}\right|=\mathfrak{c}$. Define $g:[0,1] \rightarrow[0,1]$ in the following way

$$
g(x)= \begin{cases}\Psi(x), & \text { for } x \notin M \\ y, & \text { for } x \in M_{y}\end{cases}
$$

Clearly, $g$ is equivalent to $f$ because they have the same indicatrix and $\mathcal{A}$ measurable, as

$$
\{x \in[0,1]: g(x) \neq \Psi(x)\} \subseteq M \in \mathcal{H}(\mathcal{A}) .
$$

Using exactly the same argument as in [5], one can prove the following.

Lemma 2. Let $\mathcal{A}$ be a $\sigma$-algebra containing Bor such that $\mathcal{H}(\mathcal{A})$ contains a set of size $\mathfrak{c}$. Let $f:[0,1] \rightarrow[0,1]$ be a function constant on a set of cardinality $\mathfrak{c}$. Then $f$ is equivalent to an $\mathcal{A}$-measurable function.

Theorem 3. A function $f:[0,1] \rightarrow[0,1]$ is equivalent to a Marczewski-measurable one, if and only if, either $f[[0,1]]$ contains a perfect set, or there exists $y \in[0,1]$ such that $\left|f^{-1}[\{y\}]\right|=\mathfrak{c}$. In particular, each Lebesgue measurable function is equivalent to a Marczewski-measurable one, and vice versa.

Proof. It is folklore that the algebra of Marczewski-measurable sets satisfies the assumptions of Lemma 1 and 2, which shows sufficiency of this condition.

To prove the necessity, we can assume that $f$ is itself Marczewski-measurable. Then there exists a perfect set $P$ such that $f \nmid P$ is continuous. If $f[P]$ is uncountable, then it contains a perfect set. Otherwise, there exists $y \in f[P]$ such that the set $f^{-1}[\{y\}]$ is of size continuum.

One can easily see that the argument above is more general than for Marczewskimeasurable functions. The assumptions needed for sufficiency of the characterization (i.e. the assumptions of Lemma 1 and 2) are very general (as long as the extensions of Bor are concerned). To prove the necessity, we only used the fact that a Marczewski-measurable function is continuous on a perfect set.

Let us say that a class of functions $\mathcal{F}$ from a Polish space to $[0,1]$ has the Weak Continuous Restriction Property (WCRP for short) if every $f \in \mathcal{F}$ is continuous on a perfect set. This is a weaker property than the Continuous Restriction Property considered in [6], where the perfect set is required not to belong to a given $\sigma$ ideal. It is also a weaker version of a suitable instance of the Sierpiński condition considered in [1]. 
Let us point out that some natural reformulations of the WCRP would be in fact equivalent.

Proposition 4 (folklore). The following conditions are equivalent for $f: X \rightarrow$ $[0,1]$, where $X$ is a Polish space.

(1) $f\lceil P$ is continuous, for a perfect set $P$,

(2) $f \uparrow B$ is continuous, for an uncountable Borel set $B$,

(3) $f \uparrow P$ is Borel, for a perfect set $P$,

(4) $f \nmid B$ is Borel, for an uncountable Borel set B.

As an immediate generalization of Theorem 3 we obtain the following.

Theorem 5. Let $\mathcal{A}$ be a $\sigma$-algebra of subsets of a Polish space $X$ containing $\operatorname{Bor}(X)$ such that $\mathcal{H}(\mathcal{A})$ contains a set of size $\mathfrak{c}$. Assume that the class of $\mathcal{A}$-measurable functions has WCRP. Then a function $f: X \rightarrow X$ is equivalent to an $\mathcal{A}$-measurable one, if and only if, either $f[X]$ contains a perfect set, or there exists $y \in X$ such that $\left|f^{-1}[\{y\}]\right|=\mathfrak{c}$.

Proof. Analogous to the proof of Theorem 3.

The important class of algebras satisfying the assumptions of Theorem 5 are the algebras of sets decided by popular forcing notions. We can interpret the Marczewski-measurable sets as sets decided by the Sacks forcing $\mathbb{S}$ (i.e. such sets $X$ that the set of conditions in $\mathbb{S}$ which either miss $X$ or are included in $X$ is dense). It is folklore that if we replace the Sacks forcing by the forcing notion of Laver, Mathias, Miller or Silver, the functions measurable with respect to the respective $\sigma$-algebra have WCRP. Also, each of the respective ideals ${ }^{1}$ contains a set of size $\mathfrak{c}$ (this follows from the results from [2]). In particular, in the case of Mathias forcing, we obtain the following.

Corollary 6. Let $\mathcal{A}$ be the $\sigma$-algebra of completely Ramsey subsets of $2^{\omega}$. Then a function $f: 2^{\omega} \rightarrow 2^{\omega}$ is equivalent to an $\mathcal{A}$-measurable one, if and only if, either $f\left[2^{\omega}\right]$ contains a perfect set, or there exists $y \in 2^{\omega}$ such that $\left|f^{-1}[\{y\}]\right|=\mathfrak{c}$.

\section{REFERENCES}

[1] A. Bartoszewicz and E. Kotlicka, Relationships between continuity and abstract measurability of functions, Real Analysis Exchange.

[2] J. Brendle, Strolling through paradise, Fundamenta Mathematicae 148 (1995), no. 1, 1-25.

[3] A. Komisarski, H. Michalewski, and P. Milewski, Functions equivalent to Borel measurable ones, Preprint.

[4] A. Kwiatkowska, On a continuous function taking every value a given number of times, Preprint (2005).

[5] M. Morayne and Cz. Ryll-Nardzewski, Functions equivalent to Lebesgue measurable ones, Bulletin of the Polish Academy of Sciences 47 (1999), no. 3, 263-265.

[6] I. Recław, Restrictions to continuous functions and Boolean algebras, Proceedings of the American Mathematical Society 118 (1993), no. 3, 791-796.

[7] E. Szpilrajn (Marczewski), Sur une classe de fonctions de m. Sierpiński et la classe correspondante d'ensembles, Fundamenta Mathematicae 24 (1935), 17-34.

\footnotetext{
${ }^{1}$ In case of these forcing notions, the ideal of hereditarily measurable sets coincides with the ideal of sets missed by a dense set of conditions.
} 
Institute of Mathematics, Warsaw University, ul. Banacha 2, 02-097 Warszawa, PolAND

E-mail address: mkysiak@mimuw.edu.pl 